\section{Las universidades públicas argentinas \\ y la Economía Social y Solidaria. \\ Hacia una educación democrática y emancipadora}

\author{
Rodolfo Pastore \\ Docente investigador de la Universidad \\ Nacional de Quilmes, Argentina. \\ Director del Programa de Extensión \\ Construyendo Redes Emprendedoras \\ en Economía Social (CREES); \\ de Integración social, Comunidad \\ de aprendizajes, Tecnologías para \\ la inclusión social y Arte comunitario \\ (ICOTEA) y de la Unidad Académica \\ Observatorio del Sur de la Economía \\ Social y Solidaria.
}

\section{Resumen}

Desde una experiencia universitaria singular de la Universidad Nacional

de Quilmes, el presente trabajo realiza un abordaje sobre las vinculaciones entre las universidades públicas y la Economía Social y Solidaria (ESS). Luego de una breve presentación de nuestro enfoque en ESS, el trabajo hace un paneo de los avances alcanzados en las universidades de nuestro país en docencia, extensión e investigación en la temática, concluyendo con algunos desafíos para profundizar el camino ya recorrido hacia una educación democrática y emancipadora.

\section{Palabras clave}

- Economía Social y Solidaria

- democratización universitaria

- extensión

- docencia e investigación
Economía Social y Solidaria /

Perspectivas

RECEPCIÓN: 26/06/15

ACEPTACIÓN FINAL: 27/08/15

\section{Resumo}

A partir de uma experiência universitária de singularidade da Universidade Nacional de Quilmes, o presente trabalho realiza uma abordagem sobre as ligações entre as universidades públicas e a economia social e solidária (ESS). Depois de uma breve apresentação de nossa abordagem em ESS, o trabalho realiza uma visão panorâmica dos avanços alcançados nas universidades de nosso país no ensino, extensão e pesquisa na temática, concluindo com alguns desafios para profundizar o caminho já percorrido até uma educação democrática e emancipatória.

\section{Palavras-chave}

- Economia Social e Solidária

- democratização universitária

- extensão

- ensino e pesquisa
Para citación de este artículo

Pastore, R. (2015). Las universidades públicas argentinas y la Economía Social y Solidaria. Hacia una educación democrática y emancipadora. En Revista +E versión digital, (5), pp. 20-31. Santa Fe, Argentina: Ediciones UNL. 


\section{Introducción}

En los últimos años, la ESS tiene mayor presencia en la agenda universitaria, tanto en Argentina como en otros países de América del Sur. A su vez, esa mayor significación en los ámbitos académicos acompaña el creciente reconocimiento de la temática en las políticas públicas nacionales y locales, las cuales desde hace más de una década son impulsadas por los gobiernos democráticos y populares de la región. De igual forma, más recientemente se observa cierta atención a la ESS en algunos organismos multilaterales. ${ }^{1}$

Esa mayor consideración de la ESS en la agenda académica y política, con sus avances y problemáticas, tiene como principal antecedente las respuestas sociales que desde hace más de dos décadas vienen dando diversidad de grupos y organizaciones colectivas contra la globalización neoliberal, desarrollando una pluralidad de trayectorias empíricas de economía popular, social y solidaria. Desde nuestro punto de vista, esta creciente importancia empírica de la ESS es el origen social para la mayor consideración en las otras dos dimensiones comentadas, la dimensión política (en este caso de políticas públicas) y la dimensión simbólica, que incluye en particular una cuestión clave para este trabajo, el debate y actividad académica de las universidades públicas. Sobre esa base, venimos sosteniendo en distintos trabajos ${ }^{2}$ que la ESS constituye un campo socioeconómico, simbólico y político atravesado por diversas fuerzas y relaciones de poder internas y externas a su constitución. De allí que, a nuestro entender, la ESS designa al menos esas tres dimensiones interrelacionadas:

a) Una dimensión de trayectorias empíricas de otra forma de hacer economía, que une finalidad social de reproducción de la vida con dinámicas de gestión asociativa, democrática y solidaria; la cual incluye tanto las experiencias más institucionalizadas (cooperativas y mutuales más históricas), como las trayectorias más recientes de la "economía social emergente" y las formas asociativas de la economía popular.

b) Una dimensión político-organizativa de proyectos de sociedad en disputa, tensionada entre la adaptación a las lógicas económicas hegemónicas, versus la capacidad para transformarlas en pos de profundizar la democracia y la solidaridad sistémica.

c) Una dimensión simbólica (conceptual, cultural, educativa y comunicacional) de construcción social de sentidos sobre la interacción económica, centrada en las condiciones de reproducción de la vida y en las relaciones de las personas entre sí y con su hábitat vital.

1) Tal como sucede recientemente con la Organización Internacional del Trabajo y Naciones Unidas.

2) Ver Pastore (2006, 2010 y 2014a). 3) Es así que el Comité DESC de Na- ciones Unidas declaró que la "educación es un derecho humano intrínseco y un medio indispensable de realizar otros derechos humanos", y que para ello los sistemas educativos deben
Desde este punto de vista, el fortalecimiento estratégico de la ESS requiere considerar los desafíos en clave de construcción de poder en dichas dimensiones: a) el poder material, tecnológico y socioeconómico de las experiencias de la ESS; b) el poder sociopolítico y de confluencia organizativa de la diversidad de actores y políticas vinculados a la misma; y c) la construcción social de poder simbólico y de saberes acordes con los principios de este campo en formación. En ese contexto, por convicción y experiencia propia, consideramos que la universidad pública puede contribuir a avanzar en dichos desafíos (particularmente en la dimensión simbólica), si mediante metodologías participativas y de diálogo de saberes con los actores involucrados orienta los recursos y capacidades humanas que dispone para construir conocimientos, plataformas de aprendizajes y tecnologías sociales que sirvan al despliegue de la potencia y al desarrollo de las capacidades de la ESS. De hecho, con sus avances y dificultades, éste es gran medida el intento de una diversidad de proyectos y equipos universitarios que venimos trabajando en estas cuestiones, tanto en nuestro país como en otros de América Latina. De allí que el presente trabajo se proponga plantear algunas reflexiones que esperamos puedan ser útiles para fortalecer el vínculo entre las universidades y la ESS en el camino hacia una educación democrática y emancipadora.

\section{Por una universidad orientada}

\section{a la democratización y la emancipación}

La perspectiva de una educación superior democrática y emancipadora es coherente con la impronta de la ESS y con los avances en el reconocimiento internacional de la educación como un derecho esencial de las personas. Este reconocimiento de la educación como derecho se expresa claramente en los acuerdos internacionales sobre derechos humanos, en particular como parte indisoluble de los derechos económicos, sociales y culturales (DESC). ${ }^{3}$ En la misma dirección, pero específicamente para la educación superior y universitaria, la Conferencia Regional de Educación Superior de América Latina y el Caribe, celebrada en 2008, declaró a la educación superior como un derecho humano y un bien público social que debe ser garantizado por el Estado, velando por que la misma sea pertinente y de calidad. En ese contexto, entendemos clave la contribución de las universidades públicas a la profundización democrática, contar con disponibilidad institucional, material y de recursos para garantizarlo, así como condiciones de accesibilidad, calidad, pertinencia y adecuación a las necesidades de las comunidades destinatarias". Obs. General № 13 sobre el Derecho a la Educación del Comité DESC de Naciones Unidas, párrafo $1,1999$. 
considerando dicha profundización en un doble sentido. Primero, en una acepción de democratización continua, como multiplicación de la base de ciudadanía efectiva que amplía el acceso y ejercicio pleno de derechos, no sólo civiles sino también económicos, culturales, educativos y de construcción de conocimientos; en este caso generando las condiciones para efectivizar el derecho a la educación superior para las mayorías populares y las clases trabajadoras, que históricamente se han visto limitadas cuando no directamente excluidas en el ejercicio de este derecho. Luego, el otro sentido de profundización democrática lo vinculamos a la noción de radicalización de la democracia propuesto por Boaventura de Sousa Santos (2010), como proceso por el cual se transforman relaciones basadas en desigualdad de poder en formas de vinculación que amplían los espacios de autoridad compartida y extienden los campos de deliberación democrática, en nuestro caso en el campo de la educación universitaria en sus vínculos con la ESS. ${ }^{4}$ Con esas ideas de fondo, en el presente trabajo nos proponemos reflexionar sobre los vínculos recientes entre la ESS y las universidades públicas de nuestro país relacionando nuestras consideraciones con las tres funciones sustantivas de la universidad: 1) democratización del acceso y formación universitaria; 2) centralidad de la extensión universitaria; y 3) investigación-acción para el fortalecimiento de la ESS.

\subsection{ESS, democratización del acceso y formación universitaria}

En las tres décadas de institucionalización democrática se amplió considerablemente el acceso de estudiantes al sistema universitario argentino. En efecto, mientras en 1983 había unos cuatrocientos mil estudiantes universitarios, en 2014 llegaron a más de un millón ochocientos mil. Asimismo, la cantidad de universidades públicas más que se duplicó en el mismo período. ${ }^{5}$ Pero este proceso tuvo

4) El mismo autor, en otro texto, propone algunas ideas-fuerzas para orientar una "reforma creativa, democrática y emancipadora de la universidad pública". Las mismas resuenan en nuestro trabajo y sintéticamente son: la democratización del acceso a la universidad; la centralidad de la extensión universitaria; la investigación-acción-participativa; la ecología de saberes; y el compromiso de la universidad con la escuela pública
(Sousa Santos, 2005).

5) Se pasó de 26 a 53 universidades nacionales entre 1984 y 2014 . Ver listado en http://portales.educacion. gov.ar/spu/sistema-universitario/listado-de-universidades-e-institutos

6) Por ejemplo, se reincorporaron docentes universitarios cesanteados o perseguidos por la dictadura, se reconocieron los centros de estudiantes, y se restableció la autonomía y cogobierno universitario. hitos importantes en cada década. En los '80, con la recuperación democrática, además de la normalización institucional ${ }^{6}$ se impulsó el ingreso irrestricto a la universidad, aunque la infraestructura y el presupuesto universitario no acompañaron en igual medida la masificación de la matrícula. A contrapelo de esa agenda democrática, en la década de 1990 las políticas neoliberales buscaron imponer una privatización de servicios (como forma de aumentar los ingresos de las universidades), de alentar el arancelamiento universitario, aunque sin éxito ante la resistencia de la sociedad y la comunidad universitaria, al mismo tiempo que facilitar ampliamente la apertura de universidades privadas. ${ }^{7}$ Paradójicamente, en dicho período también se crearon diez nuevas entidades universitarias nacionales, seis de ellas en el conurbano bonaerense. Si bien ello amplió el ingreso universitario a regiones y población previamente no incluidas, adquirieron mayor escala las problemáticas del desgranamiento y deserción universitaria. En contraposición a la agenda neoliberal, desde 2003 se buscó ampliar las condiciones de ingreso pero también la retención universitaria, en un contexto de políticas educativas orientadas a revalorizar la educación pública como un derecho, así como a promover la vinculación territorial de las universidades con las necesidades de sus comunidades de pertenencia. En esa dirección, por una parte, se profundizó la apertura de nuevas instituciones públicas en provincias y localidades donde no existía oferta previa -desde 2003 se crearon dieciséis universidades nacionales, seis de ellas en el conurbano bonaerense. ${ }^{8}$ Ello fue acompañado por un aumento significativo del presupuesto universitario, que prácticamente duplicó su participación en el Producto Bruto Interno. ${ }^{9}$ De igual modo, con la finalidad de aumentar el ingreso y permanencia de estudiantes universitarios de bajos ingresos, se amplió considerablemente el Programa Nacional de Becas (becas universitarias, Becas Bicentenario y articulación con el Progresar), ${ }^{10}$ incluyendo con prioridad de otorgamiento a sujetos de aprendizaje tradicionalmente excluidos
7) En efecto, entre 1990 y el 2002 se aprobaron más de veinte universidades privadas, tantas como las que se habían creado en toda la historia previa de nuestro país.

8) Las seis del conurbano bonaerense son Arturo Jauretche, Avellaneda, Moreno, Hurlingham, José C. Paz, Oeste. Las restantes: Alto Uruguay, Chaco Austral, Chilecito Comechingones, de la Defensa, de las Artes (ex IUNA), Rafaela, Río Negro, Tierra del Fuego, Villa Mercedes.
9) En efecto, según datos oficiales el presupuesto universitario nacional pasó del 0,53\% en 2003 al 1,02 del PBI en 2012. Ver SPU-ME, 2013. 10) Según la información oficial se pasó de un presupuesto nacional de programas de becas universitarias de $\$ 6$ millones en 2003 a $\$ 260$ millones en 2013; y la cantidad paso de sólo mil 600 becas en el año 2000, a 51 mil becas en el 2014. (Ver SGCPU, 2013 y 2014; Marquina y Chiroleu, 2015). 
la perspectiva de una educación superior democrática y emancipadora es coherente con la impronta de la ESS

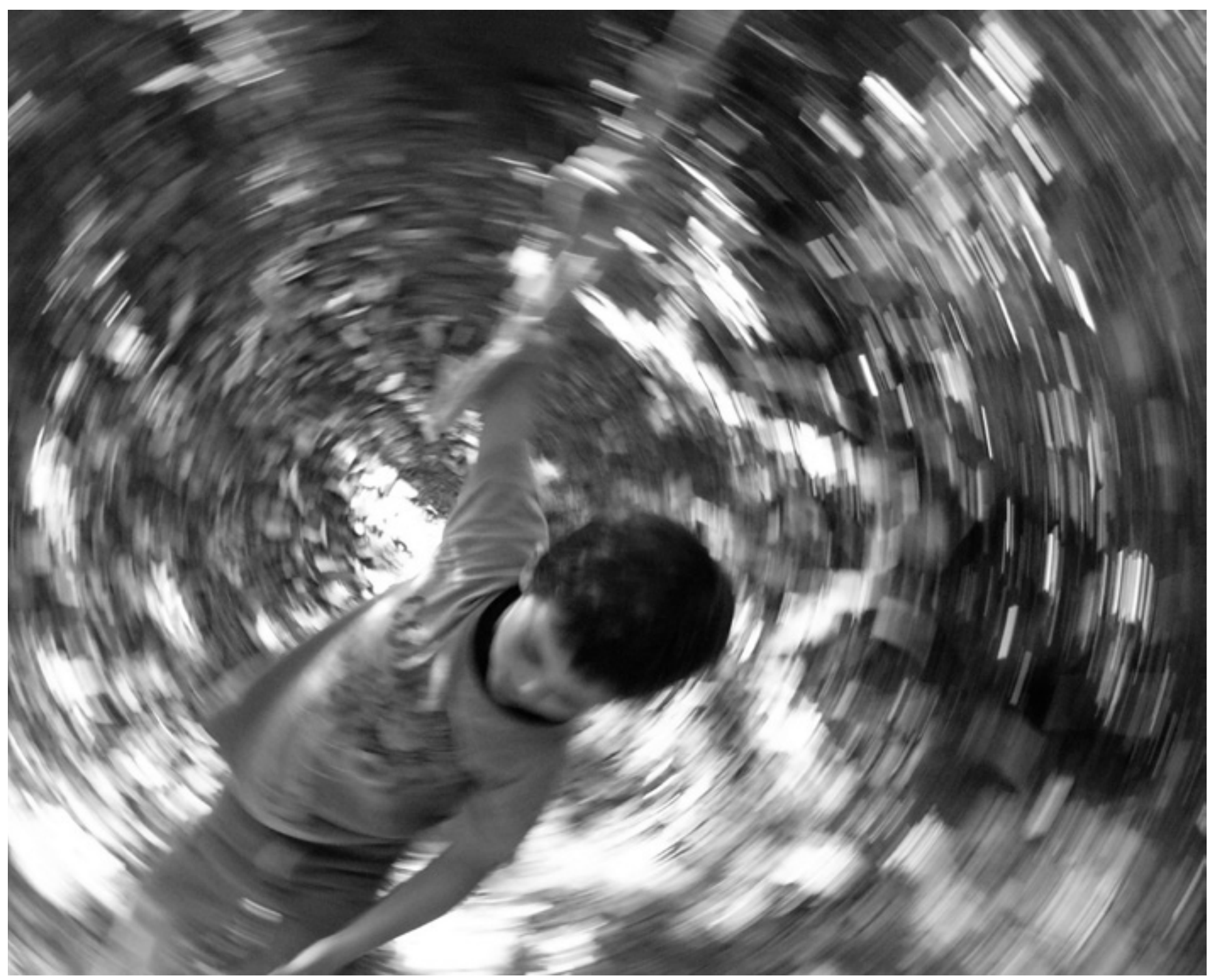


de la educación superior. ${ }^{11}$ Asimismo, se impulsaron programas complementarios para posibilitar el acercamiento de los sectores populares a la universidad ${ }^{12}$ y se buscó profundizar el programa de articulación con la educación secundaria, en un intento por facilitar el pasaje de los estudiantes entre ambos niveles. ${ }^{13}$ Todo ello ha significado un mayor acceso a la universidad por parte estudiantes provenientes de sectores sociales de ingresos medio-bajos y bajos, a tal punto que en las universidades nacionales de más reciente creación una mayoría absoluta de estudiantes (entre un 75 \% y un 95 \%) son la primera generación universitaria en su familia.

Desde nuestro punto de vista, es clave en ese contexto articular las iniciativas y sujetos de la ESS con la democratización del acceso universitario, impulsando el ejercicio efectivo del derecho a la educación superior por parte de las y los trabajadores de este campo, más aún de quienes participan en iniciativas de la economía social emergente tales como, entre otros, trabajadores vinculados a la economía popular, emprendedores de ferias y mercados de la ESS, integrantes de cooperativas de trabajo y empresas recuperadas, recicladores urbanos, productores de la agricultura familiar o promotores territoriales de microcrédito.

En tal sentido, hay algunos adelantos en esa dirección al mismo tiempo que, en perspectiva, una necesidad de ampliación y profundización. En cuanto a los avances en formación superior en ESS, lo más extendido se ha dado particularmente en dos niveles, a) en el ámbito no universitario, con las tecnicaturas superiores en economía social orientadas al desarrollo local o al rural; ${ }^{14}$ b) en el nivel de posgrado universitario, con la creación de carreras de especialización y maestrías vinculadas a la ESS. ${ }^{15}$ Pero también deben destacarse otros dos tipos de iniciativas universitarias: por una parte, las cátedras abiertas que incorporan activamente la temática de la ESS; ${ }^{16}$ por otra, las nuevas ofertas académicas de pregrado y grado universitario, en particular tecnicaturas universitarias y licenciaturas vinculadas a la temática. Las cátedras abiertas son una modalidad que por lo general permite atravesar transversalmente distintos tipos de carreras universitarias, así como en algunos casos facilitan su cursado a actores sociales sin las restricciones normativas vigentes para carreras formales. No obstante, usualmente para dichos actores no significan un trayecto formativo integral, con acreditación y validación académica sustantiva.

Esto sí ocurre con las carreras universitarias en ESS, las cuales vienen creándose en mayor medida después de la crisis de 2001 (70\%), como puede verse en el siguiente cuadro. Además, de dicho cuadro puede deducirse que las licenciaturas en su mayoría se orientan a la formación en temáticas administrativas y legales de cooperativas y mutuales, mientras que en las tecnicaturas universitarias se hace presente también la formación más amplia en ESS.

Carreras de pregrado y grado vinculadas a la ESS, 2015

\begin{tabular}{|c|c|c|c|c|c|}
\hline & $\begin{array}{l}\text { Cooperativas } \\
\text { y mutuales }\end{array}$ & ESS & Total & Hasta 2001 & Desde 2002 \\
\hline Tecnicaturas $\left(^{*}\right)$ & 2 & 3 & 5 & $20 \%$ & $80 \%$ \\
\hline Licenciaturas $\left(^{* \star}\right)$ & 4 & 1 & 5 & $40 \%$ & $60 \%$ \\
\hline Total & 6 & 4 & 10 & $30 \%$ & $70 \%$ \\
\hline
\end{tabular}

Fuente: elaboración propia sobre la base de Acosta y Levin (2013) e Informe del Senado de la Nación (2015).
(*) Tecnicaturas universitarias en: Cooperativismo (UNLP); Administración cooperativa (UPSJB); ES (UNTREF); Microcrédito y ES (UNDAV); y nuestra TUESS (UNQ) que luego comentamos. $\left(^{\star \star}\right)$ Licenciaturas en: Cooperativismo y Mutualismo (UNR, UADER, UNSE); Administración con orientación en ES (UNRN).

Desde la perspectiva enunciada de profundización democrática, creemos sustancial considerar no sólo la creación de ofertas académicas específicas, sino también otras cuestiones centrales para el debate, entre las cuales destacamos la orientación y enfoque emancipador de las mismas, la puesta en acción de pedagogías democráticas y participativas que a la vez garanticen la retención y graduación, así como las iniciativas de articulación con otros dispositivos de inclusión socioeducativa. Sostenemos estas reflexiones desde la experiencia de haber impulsado reconocidos trayectos universitarios de formación de actores de la ESS que, si bien tienen sus claroscuros (como toda práctica no ideal ni idealizada de la dimensión empírica), muestran la viabilidad y potencia de desarrollar este tipo de iniciativas. En efecto, desde el año 2010 venimos impulsado dos trayectos articulados de formación universitaria de actores en ESS (mayormente cooperativistas de trabajo, emprendedores e integrantes de organizaciones y movimientos sociales). Las mismas son:
11) Tal es el caso de perceptores de asignación universal por hijo, comunidades indígenas, padres, embarazadas, discapacidad o jóvenes vinculados a programas público de inserción laboral como "Más y mejor trabajo".

12) Entre ellos, por ejemplo, el Programa "La universidad en los barrios", donde estudiantes secundarios de barrios humildes van de visita a la universidad. También se comenzó a implementar en universidades nacionales el Programa FINES de terminalidad educativa, dirigido principalmente a jóvenes y adultos de sectores populares 13) En dicho Programa participan unas 39 universidades públicas vinculadas a unas 600 escuelas secundarias

14) Puede consultarse el Documento Base para la organización curricular las mismas en http://www.me.gov.ar/ curriform/publica/econ_socio.pdf. A diez años de esa formulación, sería de interés contar con información agregada sobre la evolución, continuidad en el tiempo y mapa actual de estas tecnicaturas en distintas jurisdicciones, así como los aportes y dificultades de estas experiencias de educación superior para la construc- ción del campo profesional en ESS. 15) Ver listado en Acosta y Levin (2013) y Senado de la Nación (2015). 16) Por ejemplo, entre otras: la Cátedra Abierta de Estudios Latinoamericanos

"José Martí" de la Universidad del Litoral; la Cátedra Abierta "Floreal Gorini" de la Universidad de Río Cuarto; o la Cátedra de Cooperativismo y ESS de la Universidad del Centro. 


\section{6}

nos interesa remarcar que la EU

es un ámbito académico

de potencialidad para impulsar

espacios de formación-acción-

reflexión con los actores territoriales

a) Diploma de Extensión Universitaria de Operador Socioeducativo en Economía Social y Solidaria (DOSESS), primer Diploma de este tipo a nivel nacional, que tiene un año de duración y no requiere título secundario para poder cursase. ${ }^{17}$

b) Tecnicatura Universitaria en Economía Social y Solidaria (TUESS), carrera universitaria de tres años de duración, que implica una democratización del acceso universitario significativo, pues para los diplomados del DOSESS que tienen secundario terminado (o lo completan mediante los programas de terminalidad educativa como el FinEs), se les valida el recorrido del diploma pues no necesitan rendir el curso de ingreso y, además, ingresan a segundo año de la tecnicatura, pues ambos planes de estudio fueron diseñados conjuntamente con esa finalidad.

Para los objetivos del presente artículo sólo señalamos unos breves apuntes sobre estas experiencias, en tanto pueden consultarse mayores referencias en otros trabajos. ${ }^{18}$ EI DOSESS y la TUESS se diseñaron simultáneamente desde la UNQ en articulación con los Ministerios de Desarrollo Social y Educación de la Nación, buscando hacer sinergia con programas públicos de promoción de cooperativas de trabajo, como es el caso del Programa Ingreso Social con Trabajo "Argentina Trabaja". Sintéticamente, representan trayectos universitarios de inclusión y transformación educativa de alto impacto, escala y cobertura territorial, que abarcaron prácticamente todo el Gran Buenos Aires ${ }^{19}$ y permitieron el acceso masivo a la educación universitaria a estudiantes trabajadores provenientes mayoritariamente de cooperativas, emprendimientos y organizaciones territoriales de la ESS. En efecto, en lo que corresponde sólo a la coordinación académica de la UNQ se han diplomado del DOSESS unos 1100 estudiantes, ${ }^{20}$ de los cuales 300 continúan cursando la TUESS, en tanto que otros 100 se han recibido en la primera camada de técnicos universitarios en ESS. Los estudiantes y egresados de ambos trayectos son mayoritariamente adultos (un $70 \%$ mayores de 30 años), así
17) La propuesta de innovación curricular del DOSESS se multiplicó, ya que no sólo otras universidades nacionales adoptaron el dispositivo de Diplomas de Extensión, sino que posteriormente también se ampliaron los diplomas en nuestra universidad en distintas temáticas, instituyéndose para ello un reglamento específico de aprobación de estos de trayectos educativos, de igual forma que sucede con las carreras universitarias.

18) Pastore (2014b), Pastore y Altschuler (2014); Pastore, Altschuler, Sena et al. (2015).

19) Por ejemplo el DOSESS se cursó en otras universidades nacionales e institutos educativos localizados en distintos territorios de Buenos Aires (los más cercanos posibles a los lugares de residencia o trabajo de los actores cursantes), que participaron también proponiendo docentes comprometidos con la propuesta.
20) Por su parte, en las otras dos Universidades Nacionales, San Martín y Moreno, que también coordinaron académicamente en sus respectivas regiones la segunda cohorte del DOSESS, egresaron un total de 773 diplomados. 


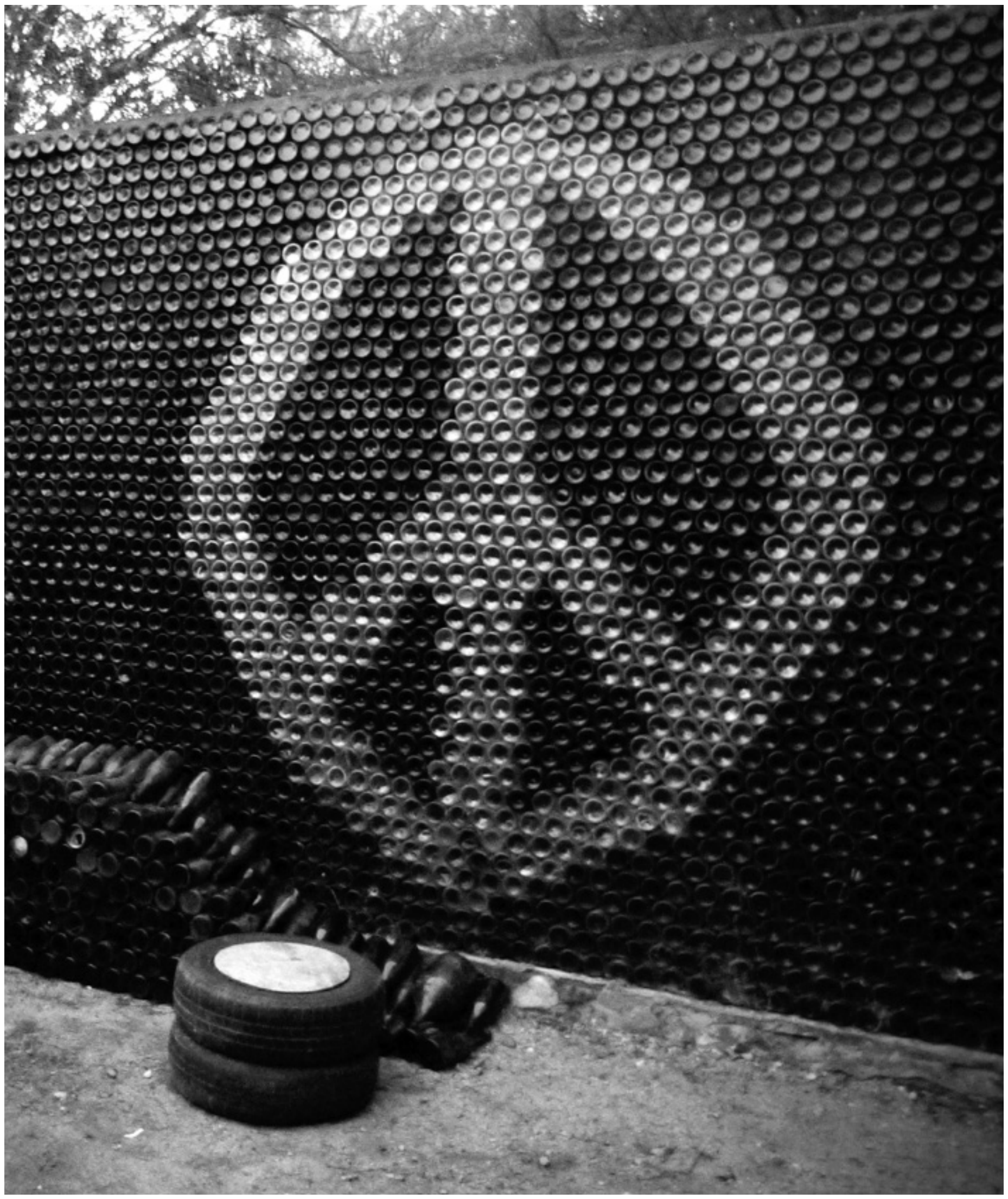


como más de dos tercios son mujeres y, buena parte de ellas, madres de familia y jefas de hogar. Por otra parte, estos trayectos universitarios registran índices educativos de retención y egreso muy elevados en comparación con otras carreras universitarias. ${ }^{21}$ Asimismo, desde su diseño e implementación pedagógica son ámbitos de reflexión-acción, educación popular, comunidad de aprendizajes y diálogo educativo, donde convergen los saberes provenientes de la experiencia y los generados en los ámbitos académicos. A su vez, en todos los casos la planificación y acción docente se trabaja en pareja pedagógica entre un docente curricular (especializado en cada espacio curricular) y un docente tutor, que principalmente cumple funciones de acompañamiento pedagógico y territorial. Esta modalidad, junto al enfoque de comunidad de aprendizajes y educación popular, tendió a garantizar esos mayores niveles de retención y terminalidad educativa, así como un enfoque académico territorial particularmente presente en las prácticas profesionalizantes, las cuales se implementan a lo largo de todos los años de formación y buscan articular los espacios curriculares con el fortalecimiento territorial de la ESS. ${ }^{22}$

En definitiva, creemos que experiencias de formación universitarias como las presentadas se posicionan desde un paradigma políticopedagógico de democratización y emancipación educativa afín con la ESS. Es decir, desde una perspectiva y práctica educativa dialógica, participativa y de ampliación derechos, intentando romper con tradiciones universitarias endogámicas y elitistas de reconocida raigambre. De allí que desde una perspectiva de ecología de saberes resulten claves también los vínculos con la Extensión Universitaria (EU).

\subsection{Centralidad de la EU en ESS}

En la última década ha crecido la importancia de la EU, tanto en la cantidad y complejidad de iniciativas universitarias de extensión, como en el mayor reconocimiento académico e institucional de la misma, aunque todavía con un peso relativo menor en comparación con las otras dos funciones sustantivas (docencia y más aún investigación). En un trabajo previo, que constituyó en 2012 el Documento Base del Congreso Argentino de Cooperativas (CAC) sobre Extensión en ESS, ${ }^{23}$ hemos sostenido que esta mayor significación de la EU reconoce tres factores principales de impulso: a) Las demandas y necesidades que se realizan a la universidad desde los actores sociales y las comunidades locales, que en el caso de la ESS se dieron particularmente desde del 2001 por parte de la economía social emergente.

b) Las iniciativas generadas desde núcleos activos de la propia comunidad universitaria; que para el caso de la ESS en sus comienzos constituyeron iniciativas un tanto aisladas de docentes o estudiantes en prácticas educativas comunitarias, voluntariados universitarios, talleres de capacitación, etc., pero que, como veremos, posteriormente alcanzaron en algunos casos mayor densidad académica e integralidad de funciones.

c) El contexto institucional y de políticas públicas de democratización y acceso a la educación superior. A lo ya señalado, conviene resaltar la incidencia de las políticas educativas que han impulsado una mayor vinculación comunitaria de las universidades, tal como sucede, entre otros casos, con los Programas Nacionales de Voluntariado Universitario, Educación Solidaria, Extensión Universitaria o Vinculación Tecnológica.

En alguna medida, esa mayor significación de la EU también se observa en relación a la ESS. ${ }^{24}$ En ese sentido, en el trabajo de referencia, pudimos relevar 76 experiencias de EU vinculadas a la ESS para 2012 que, si bien no dan cuenta del total de iniciativas existentes por entonces, representan un conjunto significativo desde el cual inferir algunas reflexiones de interés. Allí clasificamos dichas experiencias de EU según el grado de institucionalización, complejidad e integración de acciones académicas, distinguiendo cuatro tipos principales:

a) Actividades más puntuales de extensión en la temática (por lo común alguna capacitación no integrada a iniciativas más regulares). b) Prácticas socioeducativas solidarias de aprendizaje y vinculación con la comunidad (por lo general realizadas desde una cátedra docente o más recientemente desde proyectos de voluntariado universitario). c) Proyectos más institucionalizados de EU, en la mayoría de los casos designados así desde la propia experiencia.

d) Programas o unidades institucionales de mayor envergadura, expresión más institucionalizada de la universidad que articula un conjunto de iniciativas, acciones y proyectos, los cuales cubren una temática compartida y que frecuentemente integran actividades de capacitación, colaboración y acompañamiento técnico, así como prácticas socioeducativas.
21) En cuanto a la retención, en ambos casos es superior a dos tercios de los cursantes, en tanto que los índices de graduación son levemente superiores al $70 \%$ en el DOSESS y al $50 \%$ en la TUESS.
22) Puede consultarse al respecto Sansón, Sena et al. (2013); Mazzucco, Mendy, Schmalko y Toscano (2013); Pastore, Altschuler, Sena et al. (2015). 23) Ver Pastore, Baliño y Rosas (2012). 24) Éste también es nuestro propio caso como Proyecto CREES (Construyendo Redes Emprendedoras en Economía Social), que dio origen a todas nuestras iniciativas en formación universitaria (ya comentadas) o en investigación e incubación (que luego señalamos). Actualmente es un Programa de EU (CREES-ICOTEA) que integra nueve Proyectos de extensión en ESS. 
Con esos criterios, puede verse en el cuadro siguiente que, del total de experiencias relevadas, la gran mayoría de ellas corresponde a proyectos de EU (64 \%), en tanto que una docena de las mismas (13\%) representa programas o unidades institucionales de mayor amplitud. En tal sentido, una línea estratégica que podría impulsarse es el desarrollo de acuerdos interinstitucionales entre las universidades y los actores de la ESS para fortalecer los programas existentes y/o para generar nuevos, sobre todo en aquellas unidades académicas que aún no cuentan con ellos.

Tipos de iniciativas de EU en ESS, 2012

\begin{tabular}{|c|c|c|}
\hline Tipo de experiencia de EU & Total & Porcentaje \\
\hline Proyecto & 49 & 64 \\
\hline Práctica socioeducativa & 10 & 13 \\
\hline Actividades plurales & 5 & 7 \\
\hline Total & 76 & 100 \\
\hline
\end{tabular}

Fuente: Pastore, Baliño y Rosas (2012)

Por otra parte, si consideramos los tipos de emprendimientos y organizaciones de la ESS vinculados a dichas experiencias, puede observarse que una proporción ampliamente mayoritaria se vincula con experiencias de la economía social emergente mencionada previamente (86\%), más aún en el caso de las universidades del área metropolitana de Buenos Aires. Puede inducirse así la pertinencia de la vinculación prioritaria de la EU con los sectores sociales y productivos más necesitados. Al mismo tiempo, también es posible pensar una agenda estratégica para desarrollar de proyectos y/o programas universitarios que articulen ambos tipos de experiencias de la ESS (más institucionalizados y emergentes), potenciando iniciativas de cooperación intercooperativa, aprendizaje compartido, compromiso con la comunidad, educación solidaria o formación de cuadros técnico políticos.

\section{Proporción de experiencias según el tipo de ESS} al que se orienta su accionar, 2012

\begin{tabular}{|c|c|c|c|}
\hline \multirow[t]{2}{*}{ Tipo de cooperativismo al que se dirige } & \multicolumn{2}{|c|}{ Localización } & \multirow[t]{2}{*}{ Total } \\
\hline & AMBA+LP & Interior & \\
\hline ESS institucionalizada & $9 \%$ & $22 \%$ & $14 \%$ \\
\hline ESS emergente & $91 \%$ & $78 \%$ & $86 \%$ \\
\hline Total & $100 \%$ & $100 \%$ & $100 \%$ \\
\hline
\end{tabular}

Fuente: Pastore, Baliño y Rosas (2012)
Finalizando este acápite, nos interesa remarcar que la EU es un ámbito académico de potencialidad para impulsar espacios de formación-acción-reflexión con los actores territoriales. Ello no quita reconocer sus limitaciones y condicionantes, entre las cuales se encuentra su propia designación como "extensión universitaria" 25 o el riesgo de quedar marginada o "guetizada" sino que se impulsan criterios apropiados de calidad académica e interrelaciones efectivas con las otras funciones sustantivas. Precisamente desde una perspectiva académico territorial de ecología de saberes, es posible avanzar al mismo tiempo en la calidad académica de la EU y en su articulación con las demás funciones sustantivas, desde un planteo que también reconoce una "extensión" a la inversa, esto es de una "extensión" de la comunidad a la universidad. A título de ejemplo, en nuestra experiencia universitaria han sido recurrentes las actividades académicas donde los trabajadores y promotores de la ESS participan no sólo como sujetos de aprendizajes, sino sobre todo como actores claves en la construcción social de conocimiento, compartiendo y trasmitiendo sus aprendizajes y experiencias, tanto entre sí como con los demás integrantes de la comunidad universitaria. Desde esa práctica, hemos denominado a esta "extensión a la inversa" como "extensión com-universitaria" (Pastore, 2014). La misma promueve una ciudadanía universitaria activa para los actores territoriales y populares con que se vincula, oponiéndose a considerarlos como "objeto" de estudio, asistencia o transferencia; antes bien, buscando garantizar su condición como sujetos plenos de derecho, capacidades y responsabilidades. Asimismo, entendiendo que la innovación deber ser parte de la matriz identitaria de las universidades públicas, en ese accionar el status quo de la propia institución universitaria se ve interpelado por una praxis democrática y emancipadora. De allí que para expandir esa potencia de transformación universitaria es clave no recaer en perspectivas de trabajo académico unidireccionales, como puede suceder con el paradigma convencional de "transferencia" universitaria, sino, por el contrario, avanzar desde prácticas cognitivas democráticas que se basen en la ecología de saberes, la co-construcción de conocimientos, la comunicación dialógica y la interacción participativa con los actores de la comunidad, rompiendo lazos con una tendencia endogámica histórica de la institución universitaria que muchas veces deambula sobre su propia agenda. De allí la importancia de su articulación tanto con propuestas de transformación en docencia (como las comentadas) como con una agenda participativa de investigación, innovación y construcción de conocimientos socialmente pertinentes, en nuestro caso orientada al fortalecimiento del campo de la ESS, cuestión con la cual concluimos el presente trabajo. 


\section{6}

existen destacados antecedentes de Programas

de Incubadoras Universitarias en ESS en América

Latina, en particular en países limítrofes como

Uruguay o en mayor medida aún Brasil

\subsection{Investigación-acción para el fortalecimiento de la ESS}

La síntesis de conclusiones de la Comisión de Educación del mencionado Congreso de Cooperativas (CAC) declaró como desafío estratégico fortalecer el vínculo del movimiento cooperativo con las universidades, valorando para ello las tres funciones sustantivas de las mismas (docencia, extensión e investigación). Pero cabe destacar que los Documentos Base específicos del CAC para las dos últimas funciones arribaron a conclusiones diferenciadas: en un caso, como hemos visto, se observó el dinamismo de la EU en cooperativismo y ESS; en el otro, como contrapartida, se argumentó la escasa significación de estas temáticas en la agenda de investigación universitaria. En este último caso, para un período de más de una década los datos concretos contabilizaron unos 137 proyectos de investigación en ESS y cooperativismo en general, ${ }^{26}$ así como 78 proyectos de investigación específicos en ESS y cooperativismo agropecuario ${ }^{27}$ (ver cuadro posterior). Los autores señalaban la contundencia de este dato teniendo en cuenta que cada año las universidades acreditan entre cinco y siete mil proyectos de investigación ante el Programa Nacional de Incentivos a docentes investigadores. De allí que en una de las conclusiones se sostenía la necesidad de crear un fondo de financiamiento específico para investigaciones en la temática.

En tal sentido, es un hecho de significación histórica para el campo de la ESS que a partir de esos antecedentes se haya puesto en marcha desde el año 2014 el Programa de Educación en Cooperativismo y Economía Social en la Universidad, dependiente de la Secretaría de Políticas Universitarias (SPU) del Ministerio de Educación de la Nación. A tal punto que sólo en la primera convocatoria se presentaron para su evaluación 186 proyectos de investigación en ESS, de los cuales en sólo un año fueron aprobados medio centenar de proyectos de investigación aplicada o redes. ${ }^{28}$

Proyectos de Investigación Universitaria en ESS, 1996-2014

\begin{tabular}{|c|c|c|c|}
\hline $\begin{array}{l}\text { Fuente } \\
\text { de información }\end{array}$ & $\begin{array}{l}\text { Período en } \\
\text { consideración }\end{array}$ & $\begin{array}{l}\text { Cantidad } \\
\text { años }\end{array}$ & $\begin{array}{l}\text { Cantidad } \\
\text { proyectos }\end{array}$ \\
\hline Documento CAC 1: General & $1996-2011$ & 15 & 137 \\
\hline Documento CAC 2: Agropecuario & $2000-2012$ & 12 & 78 \\
\hline \multicolumn{4}{|l|}{$\begin{array}{l}\text { Programa Educación en } \\
\text { cooperativismo y ES en la }\end{array}$} \\
\hline Universidad, SPU & 2014 & 5 & 51 \\
\hline
\end{tabular}

Fuente: elaboración propia a partir de: Vuotto, Verbeke y Acosta (2013), CALISA (2012) y listado del Programa SPU.

Entre los principales sectores abordados por dichos proyectos están el cooperativismo de trabajo, residuo y recicladores urbanos, cooperativas agropecuarias, así como temáticas transversales o
26) Vuotto, Verbeke y Acosta (2013). 27) CALISA (2012)

28) Para ello se dispuso de un presupuesto específico de $\$ 10$ millones, significativamente superior al medio millón de pesos propuesto por uno de los Documento Base. 
de índole de desarrollo territorial. También puede observarse que una proporción levente superior de proyectos de investigación responde a necesidades de la economía social emergente, aunque sin tanta preponderancia como en el caso visto de EU.

Desde un punto de vista de investigación-acción, a estos avances en investigación aplicada hay que sumar dos líneas de interés en ESS la innovación tecnológica inclusiva y las incubadoras en economía solidaria. Por límites de espacio, aquí sólo podemos nombrar estas líneas, que requieren un tratamiento en mayor profundidad de análisis y debate para fortalecer el desarrollo estratégico de la ESS. Con relación a la innovación, desde el Programa Nacional de Tecnología e Innovación Social (Ministerio de Ciencia, Tecnología e Innovación Productiva) se vienen convocando durante los últimos años dos líneas de tecnologías inclusivas: a) Programa del Consejo de las Demandas de Actores Sociales (PROCODAS) desde 2008; y b) Proyectos Asociativos de Diseño (PAD) desde $2012 .{ }^{29}$ En las distintas convocatorias realizadas se han presentado entidades de la ESS en asociación con entidades científico técnicas, una parte importante de las cuales es universidad pública.

Por último, existen destacados antecedentes de Programas de Incubadoras Universitarias en ESS en América Latina, en particular en países limítrofes como Uruguay o en mayor medida aún Brasil. ${ }^{30}$ Creemos que es necesario profundizar el conocimiento de los logros y dificultades vinculadas a estas experiencias. Por nuestra parte, valorando las mismas como punto de partida para plantear estrategias de innovación que respondan a las necesidades de nuestro contexto, hemos impulsado el Programa Universitario de Incubación Social (PUIS) en ESS. ${ }^{31}$ El mismo se articula con las otras acciones académicas mencionadas, desarrolla incubación de procesos asociativos estratégicos (antes que de emprendimientos aislados) que buscan contribuir a la valorización económica y la innovación socio técnica en el campo de la ESS.

\section{Reflexiones finales}

En definitiva, hay un importante camino hecho para seguir desplegando en investigación, extensión y docencia universitaria en ESS. Asimismo, sin duda son múltiples y complejos los desafíos implicados para profundizar ese camino desde una propuesta académico-territorial fundada en la ecología de saberes, las comunidades de aprendizajes y la construcción compartida de conocimientos socialmente pertinentes. Desde ya esa construcción supone también un desafío no menor de trans-formación de las propias instituciones universitarias hacia una educación democrática y emancipadora. Un tema central en tal sentido es que dicha acción transformadora puede encontrar apoyos apreciables dentro de la comunidad universitaria (más en un contexto afín de políticas educativas), a la vez que tropezar con las inercias u oposiciones de diversos poderes instituidos. Para avanzar en ese proceso de transformación institucional y enfrentar las tendencias de institucionalización des-instituyente, creemos que,

Proyectos del Programa de Cooperativismo y ESS en las Universidades, 2014

\begin{tabular}{l} 
Sector cooperativo o de ESS \\
Cooperativas de trabajo \\
\hline Cooperativas de trabajo-textil \\
\hline Recicladores y residuos (socioambiental) \\
\hline Educación \\
\hline Salud \\
\hline Agropecuaria $\left(^{\star}\right)$ \\
\hline Crédito y consumo \\
\hline Servicios $\left(^{\star \star}\right)$ \\
\hline Transversal/territorial $\left({ }^{\star \star *}\right)$ \\
\hline Total
\end{tabular}

Fuente: elaboración propia a partir de datos del listado de proyectos aprobados del Programa SPU.

\begin{tabular}{|c|c|c|c|}
\hline$N^{\circ}$ Proyectos & Porcentaje & ES emergente & ES Institucional \\
\hline 9 & 18 & 9 & 0 \\
\hline 4 & 8 & 4 & 0 \\
\hline 6 & 12 & 6 & 0 \\
\hline 3 & 6 & 1 & 2 \\
\hline 3 & 6 & 1 & 2 \\
\hline 12 & 24 & 3 & 9 \\
\hline 2 & 4 & 0 & 2 \\
\hline 4 & 8 & 0 & 4 \\
\hline \multirow[t]{2}{*}{8} & 16 & 4 & 4 \\
\hline & & 28 & 23 \\
\hline 51 & 100 & $55 \%$ & $45 \%$ \\
\hline
\end{tabular}

() Agroecología, cultivos andinos, cunicultura, apícola, ictícola, láctea, vitivinícola. (**) Electricidad, telecomunicaciones, turismo.

$\left.{ }^{* \star \star}\right)$ Redes, comercialización, mapeos, gestión, tecnologías.

29) El financiamiento de estos programas (PAD y PROCODAS) ha crecido sig nificativamente en los últimos años (de unos $\$ 500$ mil cada uno en 2012 , paso a tres millones el PROCODAS y a un mi-
Ilón el PAD en 2015); si bien aún siguen siendo de menor magnitud en relación a otras convocatorias del MINCyT.

30) Actualmente existen en Brasil más de cien incubadoras universitarias en economía solidaria, las cuales están asociadas a dos grandes redes: a) la Red Universitaria de Incubadoras Tecnológicas de Cooperativas Populares; y b) la Red de Incubadoras de Empren- dimientos Solidarios de Unitrabalho. 31) Ver: http://www.unq.edu.ar/ secciones/384-programa-universitario-de-incubación-social-en-economía-social-y-solidaria/ 
entre otras cuestiones, es clave generar espacios de visibilidad y presencia activa de los actores de la ESS en el espacio universitario, buscando al mismo tiempo impulsar la participación plural de la comunidad universitaria (docentes, graduados, estudiantes, funcionarios y trabajadores de apoyo); así como avanzar, en la medida de lo posible, en el trabajo interdisciplinario y en la articulación integral de las tres funciones universitarias sustantivas.

\section{Referencias bibliográficas}

Acosta, M. C y Levín, A. (2013). La formación universitaria en cooperativismo y economía social en Argentina. Documento 80 del Centro de Estudios de Sociología del Trabajo. Buenos Aires: Facultad de Ciencias Económicas. Universidad de Buenos Aires. CALISA (2012). El cooperativismo agropecuario y la Economía Social en las Universidades Públicas". Documento Base para el debate del Congreso Argentino de Cooperativas (CAC), eje temático Universidad y cooperativismo. Cátedra Libre de Soberanía Alimentaria. Facultad de Agronomía, Universidad de Buenos Aires. Recuperado de: http://www.agro.uba.ar/content/el-cooperativismo-agropecuario-y-la-econom-social-en-las-universidades-p-blicas-calisa-2012 (consulta 7 de mayo de 2015).

Freire, P. (1998). ¿Extensión o comunicación? La concientización en el medio rural. México: Siglo XXI.

Marquina, M. y Chiroleu, A. (2015). ¿Hacia un nuevo mapa universitario? La ampliación de la oferta y la inclusión como temas de agenda de gobierno en Argentina. En revista Propuesta educativa $N^{\circ} 43$, Dossier, Políticas de Educación Superior en América Latina: las agendas del siglo XXI. Buenos Aires: FLACSO-Argentina. Recuperado de: http://www.propuestaeducativa.flacso.org.ar/dossier. php?num=43 (consulta 7 de mayo de 2015).

Pastore, R. (2006). Diversidad de trayectorias, aproximación conceptual y pluralidad de proyectos de la Economía Social. En Documento 54 del Centro de Estudios de Sociología del Trabajo. Buenos Aires: Facultad de Ciencias Económicas, Universidad de Buenos Aires.

Pastore, R. (2010). Un panorama del resurgimiento de la economía social y solidaria en la Argentina. En Revista de Ciencias Sociales 2(18), primavera 2010. Bernal: Universidad Nacional de Quilmes.

Pastore, R. (2014a). La economía social y solidaria, una construcción colectiva y plural. En Lozano, M. y Flores, J. (coords.). Democracia y sociedad en la Argentina contemporánea. Reflexiones sobre tres décadas. Bernal: Universidad Nacional de Quilmes.

Pastore, R. (2014b). Construyendo espacios universitarios de formación de actores de la economía social y solidaria. Reflexiones desde una práctica académica-territorial. En: Voces en el Fénix (38), Historias Extraordinarias. Buenos Aires: Facultad de Ciencias Económicas, Universidad de Buenos Aires.

Pastore, R. y Altschuler, B. (2014). Economía social y solidaria: un campo socioeconómico, simbólico y político en construcción. Miradas y prácticas desde la Universidad pública. En Fidel, C. y Villar, A. (coords.). Miradas y controversias del desarrollo territorial en Argentina. Aproximación a un enfoque analítico. Buenos
En ese intento estamos empeñados un conjunto de equipos universitarios en esta temática, y recientemente más de veinticinco de los mismos comenzamos a confluir en un espacio universitario en ESS vinculado a la Red de Extensión de las Universidades Nacionales (REXUNI). Es mucho lo que hay que seguir construyendo pero, con sus avances y dificultades, no es poco el camino ya recorrido para continuar apostando a la profundización democrática universitaria desde la ESS.

Aires: Centro Cultural de la Cooperación - UNQ

Pastore, R.; Altschuler, B; Sena, S. et al. (2015). Sistematizando prácticas en economía social y solidaria: universidad y empresas sociales para un desarrollo territorial inclusivo. En: Fidel, C. y Villar, A. (coords.). Miradas y controversias del desarrollo territorial en Argentina. Aproximación a un enfoque analítico. Buenos Aires: Centro Cultural de la Cooperación - UNQ.

Pastore, R.; Altschuler, B; Sena, S. y Schmalko, N. (2012). Formación para la Autogestión colectiva en articulación con políticas públicas de inclusión social. En Grellier, H.; Gago, M. \& Arando, S. (coords.). La Economía Social y Solidaria: ¿un paradigma más actual que nunca? España: Mondragon Unibertsitatea, Colección BILDUMA.

Pastore, R.; Baliño, G. y Rosas, G. (2012). La extensión universitaria vinculada a organizaciones cooperativas y de economía social y solidaria. Algunas reflexiones para el debate. Documento base para el debate, Congreso Argentino de Cooperativas (CAC), eje temático Universidad y cooperativismo. Recuperado de: http:// www.cac2012.coop/?page_id=112 (consulta 7 de mayo de 2015)

Senado de la Nación (2015). Formación con títulos terciarios, de grado y posgrado en la temática de la Comisión. Documento de la Comisión de economías regionales, economía social, micro, pequeña y mediana empresa. Recuperado de: http:// www.senado.gov.ar/prensa/12234/noticias (consulta 7 de mayo de 2015).

Secretaría de Gestión y Coordinación de Políticas Universitarias (2013). Informe de gestión 2013. Documento Secretaría de Gestión y Coordinación de Políticas Universitarias, Ministerio de Educación de la Nación. Recuperado de: http://informacionpresupuestaria.siu.edu.ar/DocumentosSPU/informe_de_gestion_2013/ INFORME_DE_GESTION_2013.pdf (consulta 7 de mayo de 2015). SPU. Secretaría de Gestión y Coordinación de Políticas Universitarias (2014). Informe de gestión 2014. Documento Secretaría de Gestión y Coordinación de Políticas Universitarias, Ministerio de Educación de la Nación. Recuperado de http://informacionpresupuestaria.siu.edu.ar/DocumentosSPU/INFORME_GESTION_2014.pdf (consulta 7 de mayo de 2015).

Sousa Santos B. de (2010). Refundación del Estado en América Latina. Perspectivas desde una epistemología del Sur. Lima, Perú: Instituto Internacional de Derecho y Sociedad. Programa Democracia y Transformación Global.

Vuotto, M.; Verbeke, G; Acosta, M.C. (2013). La investigación en cooperativismo en instituciones universitarias argentinas. En Documento 81 del Centro de Estudios de Sociología del Trabajo. Buenos Aires: Facultad de Ciencias Económicas, Universidad de Buenos Aires. 